disease inactivation are scarce. Gene expression profiling analysis could be useful to elucidate the pathogenic mechanisms of diseases, and differential gene expression analysis between diverse disease conditions produces gene signatures characteristic of the state or disease being studied. Objectives: Our aim was to compare the transcriptional profiles of patients with clinically active versus inactive (remission state) PsA (peripheral joint subset), and healthy controls (HCs).

Methods: From a cohort of around 300 patients affected by PsA according to CASPAR criteria, we first selected 20 patients (peripheral arthritis subset) with active disease state (without biologic treatment ongoing) (A) and 20 patients with $>1$-year remission induced by TNF $\alpha$ antagonism $(R)$, as assessed by DAPSA $>14$, and DAPSA $\leq 4$ scores respectively, and from $20 \mathrm{HCs}$ matching for age and gender ratio. Both PsA groups were not on corticosteroid treatment. RNA from peripheral blood was extracted and, following quality analysis by Agilent Bioanalyzer, each condition has been profiled using RNAs pools in biological duplicates by distinct Affymetrix Human GeneChip HTA 2.0, for a total of 6 arrays. Data analysis was performed using the commercial software Partek Genomics Suite, V 6.6. To identify a transcript as differentially expressed, a value of fold change 1.5 and $p$-value 0.05 has been set.

Results: The Venn diagram shows all comparative groups (A vs R, A vs $\mathrm{HC}, \mathrm{R}$ vs $\mathrm{HC}$ ) with their relative amount of transcripts differentially expressed, generated using abovementioned parameters, and the relationship between sets (fig1, panel A). Using the list of transcripts differentially expressed in at least one of the aforementioned comparison, a hierarchical clustering was carried out to highlight the intra-condition expression profile. We have identified (arbitrarily) 4 clusters of transcripts with analogous transcriptional profile and to each of them a color code has been assigned (Heatmap in Fig1, panel B). For these clusters and for all lists of transcripts differentially expressed founded by our comparative study, we carried out the Gene Set Enrichment Analysis by Gene Ontology (GO), in order to identify how molecular functions, cellular components or biological processes occurs more frequently than expected in a reference list of transcripts.

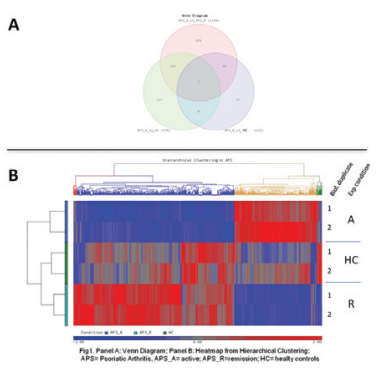

Conclusion: Observing the amount of differentially expressed transcripts, is evident that while active disease state (A) has a clear-cut different profile, the drug-induced remission $(R)$ is more similar with HCs condition. However, in the hierarchical clustering this trend of similarity does not appear in all clusters of transcripts, as shown particularly in the red, orange and green clusters. Again, the Gene Set Enrichment Analysis Score showed us that mRNA transcripts dysregulated in the $\mathrm{R}$ condition vs HCs, are involved in several biological processes related to immune system, development, response to stimulus, localization and others. Our next step will be to validate, by Real Time PCR in a large cohort of patients, the most interesting dysregulated genes covering biological functions eventually sustaining subclinical activity in PsA.

Disclosure of Interests: None declared

DOI: 10.1136/annrheumdis-2019-eular.6936

\section{FRI0358 ROLE OF MIRNA-21-5PAS A POTENTIAL BIOMARKER FOR THE INFLAMMATION PATHWAY IN PSORIATIC DISEASE AND RESPONSE TO METHOTREXATE TREATMENT}

Rohan Machhar $^{1}$, Remy Pollock ${ }^{1}$, Justine $\mathrm{Ye}^{1}$, Vinod Chandran ${ }^{1,2,3,4}$, Dafna D. Gladman ${ }^{1,2}$. ${ }^{1}$ Krembil Research Institute, University Health Network, Psoriatic Arthritis Program, Centre for Prognosis Studies in the Rheumatic Diseases, Toronto, Canada; ${ }^{2}$ Faculty of Medicine, University of Toronto, Division of Rheumatology, Toronto, Canada; ${ }^{3}$ University of Toronto, Department of Laboratory Medicine and Pathobiology, Toronto, Canada; ${ }^{4}$ Memorial University of Newfoundland, Faculty of Medicine, Newfoundland, Canada

Background: Psoriatic arthritis (PsA) is an inflammatory arthritis occurring in patients with psoriasis. Several studies have shown links between altered miRNA expression with the pathogenesis of several autoimmune disorders. We previously demonstrated that miR-21-5p was upregulated in PsA compared to psoriasis without arthritis $(\mathrm{PsC})$ and healthy controls $(\mathrm{HC})$ and is thus a potential biomarker for PsA.

Objectives: 1) To determine whether miR-21-5p is differentially expressed in PsC patients who convert to PsA vs non-convertors and validate the previous results in an independent cohort. 2) To determine the role of miR-215p in the response to methotrexate treatment (MTX) 3) To determine whether miR-21-5p modulates inflammation in psoriatic disease.

Methods: Serum \& whole blood RNA samples were collected from 54 convertors and 54 non- convertors (matched for age, sex, psoriasis duration), 40 patients with early PsA (<2 years' disease duration and not receiving biologic therapy), 40 patients with $\mathrm{PsC}$ (>10 years disease duration, not receiving biologic therapy, and matched to PsA patients on age, sex, psoriasis duration, and age of psoriasis onset), and $40 \mathrm{HC}$ (matched to patients based on age, sex). RNA was extracted using the Tempus Spin RNA Isolation Kit. miR-21-5p was validated using droplet digital PCR (ddPCR). Serum II-17, CXCL10, IL-23, TGF $\beta 1$ were measured by commercially available ELISA kits. Mann- Whitney test, Kruskal-Wallis test, Wilcoxon signed rank test and Spearman correlations were performed.

Results: The expression of miR-21-5p was significantly higher in convertors compared to non-convertors (Fold change $(F C)=2.16, \quad p=0.002$ ). miR21-5p was upregulated in PsA compared to $\mathrm{PsC}(\mathrm{FC}=2.32, \mathrm{p}=0.001)$ and $\mathrm{HC}(\mathrm{FC}=15.7, \mathrm{p}=<0.0001)$ in the validation cohort. miR-21-5p was significantly down regulated 24 weeks post-MTX treatment in $30 \mathrm{PsA}$ patients $(F C=1.9, p<0.008)$, which correlated with swollen $(r=0.49, p=0.003)$ and tender joint counts $(r=0.41, p=0.02)$ supporting a possible role in inflammation pathway in PsA patients. IL-17 levels in PSA \& PsC were significantly higher from $\mathrm{HC}(p=0.03)$, but not different between PsA \& PsC. IL-17 levels were down- regulated post MTX treatment and correlated with miR-21-5p $(r=0.56, p=<0.001), C X C L 10$ levels $(r=0.48, p=0.01), \quad I L-23$ levels $(r=0.43, p=0.02)$, TGF $\beta 1 \quad(r=-0.45, p=0.04)$ and total active joint counts $(r=0.40, p=0.016)$.

Conclusion: Up regulated expression levels of miR-21-5p in convertors and in PsA patients suggests a role as a potential biomarker for PsA. In the presence of upregulated miR-21-5p, IL-17 and IL-23 are upregulated while TGF $\beta 1$ is down regulated. When miR-21-5p is decreased IL-17 and IL-23 downregulate, with up regulation of TGF $\beta 1$. We have thus determined the role of miR21-5p as a potential biomarker for inflammation pathway in psoriatic disease and response to MTX possibly through mod ulation of CXCL10 and IL-17/IL-23 axis.

Disclosure of Interests: Rohan Machhar: None declared, Remy Pollock: None declared, Justine Ye: None declared, Vinod Chandran: None declared, Dafna D Gladman Grant/research support from: AbbVie, Amgen, Celgene, Lilly, Novartis, Pfizer, and UCB, Consultant for: AbbVie, Amgen, BMS, Celgene, Galapagos, Gilead, Janssen, Lilly, Novartis, Pfizer, and UCB

DOI: 10.1136/annrheumdis-2019-eular.7869

\section{FRI0359 KRT77 DELETIONS IN FAMILIAL INFLAMMATORY BOWEL DISEASE MAY OFFER A NOVEL LINK TO FAMILIAL SPONDYLOARTHROPATHY}

Antonio Julià ${ }^{1}$, Richard Cuthbert ${ }^{2}$, Anton Glueck ${ }^{3}$, Javier Gisbert ${ }^{4}$ Eugeni Domènech ${ }^{5}$, Maria Lopez Lasanta ${ }^{1}$, Núria Palau ${ }^{1}$, Helena Marzo-Ortega ${ }^{6}$, Dennis McGonagle ${ }^{7}$, Sara Marsal ${ }^{1} .{ }^{1}$ Vall Hebron Hospital, Barcelona, Spain; ${ }^{2}$ Leeds Biomedical Research Centre and Leeds Institute of Rheumatic and Musculoskeletal Medicine (LIRMM), Leeds, United Kingdom; ${ }^{3}$ Novartis Institutes for BioMedical Research, Basel, Switzerland; ${ }^{4}$ Hospital Universitario La Princesa, Madrid, Spain; ${ }^{5}$ Hospital Universitari Germans Trias i Pujol, Badalona, Spain; ${ }^{6}$ University of Leeds, Leeds, United Kingdom; ${ }^{7}$ University of Leeds, NIHR Leeds Musculoskeletal Biomedical Research Unit, Leeds, United Kingdom

Background: Inflammatory bowel disease, either clinical or subclinical is a cardinal pathological process that is central to the spondyloarthropathy $(\mathrm{SpA})$ category of disease that also includes psoriasis, psoriatic arthritis, uveitis and ankylosing spondylitis.

Objectives: To look for novel genes in familial $\mathrm{SpA}$ in large multcase families using a whole genome sequencing approach.

Methods: We used Whole Genome Sequencing to screen SpA family members with different disease manifestations. We screened 5 multicase SpA families (up to 8 members) with different disease manifestations. Results: We found a 1.5MB deletion of the KRT77 gene in 3 of 7 families that segregated with SpA phenotype. Given that KRT77 is a barrier protein and given that tagging SNPS from GWAS studies do not cover the region, and given that both $I B D$ and psoriasis are associated with barrier defects, we looked for an association with KRT77 deletion in 
1,000 cases of Psoriasis, 1,000 Crohn's disease (CD) and 1,001 healthy controls. Deletion genotyping was performed using TaqMan RealTime-PCR assays. The deletion genotype frequencies in controls did not deviate from Hardy-Weinberg Equilibrium $(\mathrm{p}>.1)$. We found an association between KRT77 deletion and $C D$ risk $(p=0.03)$ and also a putative association with pustular psoriasis $(p<0.05)$. We also demonstrated that the KRT77 protein is expressed in the intestinal lumen and Peyers Patches in addition to the know skin expression.

Conclusion: Given the strong association between SpA and subclinical gut inflammation and barrier defects in IBD these findings suggest that KRT77 heterozygous deletions with dysregulated gut permeability may play a role in IBD and the wider SpA phenotype.

Acknowledgement: We would like to thank the clinical researchers and patients participating in the IMID Consortium for their collaboration Disclosure of Interests: Antonio Julià: None declared, Richard Cuthbert: None declared, Anton Glueck: None declared, Javier Gisbert: None declared, Eugeni Domènech: None declared, Maria Lopez Lasanta: None declared, Núria Palau: None declared, Helena Marzo-Ortega Grant/ research support from: Janssen, Novartis and Pfizer, Consultant for: AbbVie, Celgene, Janssen, Eli-Lilly, Novartis and UCB, Speakers bureau: AbbVie, Celgene, Janssen, Eli-Lilly, Novartis and UCB, Dennis McGonagle Consultant for: Lilly, Novartis UCB, Speakers bureau: Lilly, Novartis UCB, Sara Marsal: None declared

DOI: 10.1136/annrheumdis-2019-eular.7745

\section{FRI0360 ANALYSIS OF BLOOD MONOCYTE TRANSCRIPTOMES AND BONE MARROW SAMPLES OF PATIENTS WITH AXIAL SPONDYLOARTHRITIS REVEALS THEIR CHANGES RELATED TO ACTIVATION AND MYELOPOIESIS}

Fabian Karow ${ }^{1}$, Joachim Grün${ }^{2}$, Biljana Smiljanovic ${ }^{2}$, Denis Poddubnyy ${ }^{1}$, Ulrike Erben ${ }^{1}$, Elisabeth Kenngott ${ }^{1}$, Thomas Häupl ${ }^{2}$, Andreas Grützkau²,

Uta Syrbe $1 .{ }^{1}$ Charite, Berlin, Germany; ${ }^{2}$ Deutsches Rheumaforschungszentrum, Berlin, Germany

Background: Axial spondyloarthritis (axSpA) is characterized by inflammation within the axial skeleton. Previously, we observed preactivation of circulating monocytes of axSpA patients suggesting systemic immune activation apart from local skeletal inflammation.

Objectives: The aim of this study was to characterize systemic immune activation in axSpA by analyzing axSpA-specific monocyte transcriptomes and changes in myelopoiesis.

Methods: We performed transcriptomic analyses using GeneChip HGU133 Plus2.0 arrays of CD14+ monocytes isolated from peripheral blood of 25 HLA-B27+ axSpA patients and 10 healthy controls (HC). 10 patients were classified as non-radiographic axSpA (nr-axSpA) and 15 patients as ankylosing spondylitis (AS). Robust Multichip Averaging Algorithm normalization and high performance chip data analysis were used to select differentially expressed transcripts that define 1) axSpA-geneexpression profile and 2) profile that discriminates $\mathrm{nr}$-axSpA from AS patients. Functional analysis of significant IDs was performed with Gene ontology $(\mathrm{GO})$ and reference transcriptomes that portrayed myelopoiesis in bone marrow and monocytes activation with TNF, LPS, IFN and G-CSF. Myelopoiesis in bone marrow was studied by immunohistology according to MPO and CD15 expression in sections from facet joints of AS patients and controls (CO) without skeletal disease ( $n=6 /$ group).

Results: Comparison between axSpA and HC monocyte transcriptomes identified 957 differentially expressed transcripts that allowed separation of both groups by hierarchical clustering. GO analysis revealed associations of axSpA related transcripts with cell-cell adhesion, immune responses including Ras-signalling, Jak-STAT-signalling, innate immunity. Comparison of axSpA related transcripts with reference transcriptomes identified overlap with late myelopoiesis, pronounced mobilisation of monocytes from bone marrow into blood triggered by G-CSF and weak TNF/LPS activation.

$\mathrm{Nr}$-axSpA and AS monocytes differed in 562 transcripts. GO analysis of these transcripts suggested alterations in mitochondrial activity, peptide transport and DNA repair. According to reference transcriptomes, profiles of nr-axSpA monocytes differed from those in AS by gene patterns of late myelopoiesis and G-CSF triggered mobilisation from bone marrow.

In histological analyses of bone marrow a significantly higher percentage of MPO+ cells indicative of left shifted granulopoiesis was found in AS vs. CO facet joints $(p<0.01)$.

Conclusion: AxSpA monocyte transcriptomes reflect weak immune activation by cytokine and/or LPS and premature release from bone marrow into blood. Bone marrow analysis shows a left shifted granulopoiesis in
AS - both of which may contribute to pathological immune response within the skeleton in axSpA.

\section{REFERENCE:}

[1] Conrad K, Wu P, Sieper J, Syrbe U. In vivo pre-activation of monocytes in patients with axial spondyloarthritis. Arthritis Res Ther. 2015 Jul 16:17:179. Doi: 10.1186/s13075-015-0694-2. PubMed PMID: 26178906

Disclosure of Interests: Fabian Karow: None declared, Joachim Grün: None declared, Biljana Smiljanovic: None declared, Denis Poddubnyy Grant/research support from: AbbVie, Merck Sharp \& Dohme, Novartis, Consultant for: AbbVie, Bristol-Myers Squibb, Janssen, Merck Sharp \& Dohme, Novartis, Pfizer, UCB Pharma, Speakers bureau: AbbVie, BristolMyers Squibb, Janssen, Merck Sharp \& Dohme, Novartis, Pfizer, Roche, UCB Pharma, Ulrike Erben: None declared, Elisabeth Kenngott: None declared, Thomas Häupl: None declared, Andreas Grützkau: None declared, Uta Syrbe: None declared

DOI: 10.1136/annrheumdis-2019-eular.8051

\section{FRI0361 INNATE VERSUSADAPTIVE IL-17A PRODUCING CELLS IN AXIAL SPONDYLOARTHRITIS}

nicolas rosine ${ }^{1}$, Surya Koturan ${ }^{1}$, Hanane Yahia ${ }^{1}$, Claire Leloup ${ }^{1}$, Elisabetta Bianchi ${ }^{1,2}$, Corinne Miceli Richard ${ }^{1,2} 3^{2}$, Lars Rogge ${ }^{1,2}{ }^{1}{ }^{1}$ Institut Pasteur, Immunoregulation unit, Immunology department, Paris, France, ${ }^{2}$ Unité Mixte de Recherche, Institut Pasteur/AP-HP Hôpital Cochin, Paris, France; ${ }^{3}$ Hôpital Cochin. Assistance Publique - Hôpitaux de Paris, Department of Rheumatology, Paris, France

Background: IL-17A-inhibition has been very successful in AxSpA but the cell populations targeted by this new therapeutic remain unknown. This question is relevant because the recent failure of anti-IL-23 in AxSpA demonstrated that IL-23-independent IL-17-producing cells may be of particular relevance for $\mathrm{SpA}$ pathogenesis. Some data from literature sug gests the involvement of MAIT, $\gamma \delta \mathrm{T}$, and neutrophils as IL-17A producing cells in $\mathrm{AxSpA}(1-3)$. However, even though they may be responsible for IL-17A-mediated inflammation, it is still unclear which is the major IL-17A-producing cell population in this disease.

Objectives: To assess and compare gene expression profiles of neutrophils, MAIT, $\gamma \delta, \mathrm{CD} 4+$ and $\mathrm{CD} 8+\mathrm{T}$ cells from AxSpA patients.

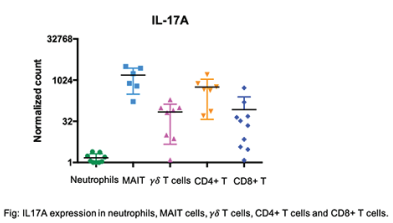

Methods: We recruited 5 healthy donors and 10 patients with a diagnosis of AxSpA according to the ASAS criteria. We compared the gene expres sion profiles of 5 sorted cell populations: 3 innate cell populations (neutrophils, MAIT and $\gamma \delta \mathrm{T}$ cells) and 2 adaptive cell populations (CD4+T and CD8+T) after cell stimulation by PMA + A23187 (calcium ionophore) $+\beta 1,3$ glucan (extracted from Aspergillus fumigatus hyphae). Published data suggested that neutrophils stimulation by Aspergillus fumigatus induces IL-17A production by these cells(4). For each of these cell populations, cytokine production and the expression of a panel of 755 genes (Autoimmune discovery panel from Nanostring including 43 genes for which a polymorphism was associated with AS) were assessed. Patient and control groups were compared with a Mann-Whitney test and com parison of cell populations was performed by a multigroup comparison. Results: There was no significant difference between patients and controls regarding gene expression profile of neutrophils, $\gamma \delta \mathrm{T}, \mathrm{CD} 4+\mathrm{T}$ and $\mathrm{CD} 8+\mathrm{T}$. We observed that 34 genes were differentially expressed between patients and controls in MAIT cells $(p=0.03, q=0.1)$. In particular, $\mathrm{T}$ cell activation genes (TBX21, $A H R, Z A P 70)$ and cell interaction genes (ITAG6, CTNNB1, ICAM2, ITGB2, SELL) were decreased in patients. Among AxSpA patients, MAIT cells were those significantly showing the highest level of IL-17A expression. IL23R and RORC were also more expressed by MAIT compared to others cell populations. IL 17A expression was very low in neutrophils but we observed that 18 out of the 43 AS associated genes were mainly expressed by neutrophils ( $p$ 